\title{
Inhibition of Poly(ADP-Ribose) Polymerase-1 Protects Chronic Alcoholic Liver Injury
}

\author{
Yanqing Zhang, ${ }^{* \dagger}$ Cheng Wang, ${ }^{*}$ Yunli Tian, ${ }^{\star \ddagger}$ Fengxiao Zhang, ${ }^{* \ddagger}$ Wenjing Xu, ${ }^{* \ddagger}$ Xiangrao Li, ${ }^{*}$ Zhiping Shu, ${ }^{* \ddagger}$ Yan Wang, ${ }^{* \ddagger}$ \\ Kai Huang, ${ }^{*}$ and Dan Huang ${ }^{*}$
}

From the Clinical Center for Human Gene Research, ${ }^{*}$ and the Departments of Geriatrics ${ }^{\dagger}$ and Cardiovascular Diseases, ${ }^{\ddagger}$ Union Hospital, Tongji Medical College, Huazhong University of Science and Technology, Wuhan, China

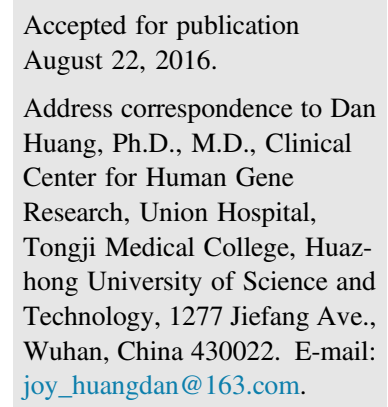

\begin{abstract}
Activation of Kupffer cells (KCs) by gut-derived endotoxin plays a pivotal role in the pathogenesis of alcoholic liver diseases (ALD). Limiting the activation of resident KCS attenuates chronic ethanolinduced liver steatosis and injury. Poly (ADP-ribose) polymerase (PARP)-1 is suggested to play a role in a number of chronic inflammatory diseases. In this study, we found a significant increase of hepatic PARP activity in mice with short-term and long-term ethanol-induced ALD. Male mice on a long-term ethanol diet exhibited severe hepatic steatosis and apoptosis and enhanced KC activation and neutrophil infiltration. However, pharmacologic inhibition of PARP activity or genetic depletion of PARP1 significantly attenuated these detrimental effects in vivo. We found that inhibition of PARP1 effectively reduced hepatic expression of genes involved in lipogenesis and elevated hepatic expression of genes involved in lipolysis. Moreover, limited $\mathrm{KC}$ activation and neutrophil infiltration were observed in PARP1 knockout mice or PARP inhibitor-treated mice. Furthermore, in vitro experiments found that LPS-induced macrophage activation was limited by PARP inhibitor, and exposure of ethanol-treated hepatocytes to this conditioned medium further decreased the number of apoptotic and steatotic cells. Taken together, these findings suggest that PARP1 inhibition protects against long-term ethanolinduced liver injury, as indicated by limited hepatocytes steatosis, apoptosis, inflammation levels, and neutrophil infiltration, mainly by limiting KC activation during the initiation of ALD. (Am J Pathol 2016, 186: 3117-3130; http://dx.doi.org/10.1016/j.ajpath.2016.08.016)
\end{abstract}

Alcoholic liver disease (ALD) is a spectrum of clinical diseases ranging from fatty liver, hepatic inflammation, and hepatitis to progressive fibrosis and cirrhosis. ${ }^{1}$ The latest surveillance report of the National Institute on Alcohol Abuse and Alcoholism found that liver cirrhosis was the 12th leading cause of death in the United States, with $48 \%$ of cases being ethanol related. Long-term ethanol consumption enhances gut permeability to bacterial lipopolysaccharide (LPS) and increases the concentration of endotoxin in the hepatic portal vein. ${ }^{2}$ Endotoxin derived from intestine activated Kupffer cells (KCs) by binding to Toll-like receptor (TLR)-4 and subsequently induced proinflammatory cytokines production in an NF- $\kappa \mathrm{B}$-dependent pathway. ${ }^{3}$ Koop et $\mathrm{al}^{4}$ found that destruction of $\mathrm{KCs}$ with $\mathrm{GdCl}_{3}$ prevented the development of hepatic steatosis and inflammation in longterm ethanol-fed mice models. Recently, medical research has identified that the activation of KCs plays a central role in the initiation of $\mathrm{ALD}$, and limiting $\mathrm{KC}$ polarization into the proinflammatory M1 phenotype is considered a promising therapeutic strategy. ${ }^{2,5-8}$ Long-term ethanol-fed mice treated with the cannabinoid $\mathrm{CB} 2$ receptor agonists, which drives hepatic M1 gene expression decreased without affecting M2 profile, significantly reduced hepatocyte steatosis and improved survival. ${ }^{6}$ Furthermore, favoring alternatively activated M2 KCs protects against ALD by targeting apoptosis of M1 KCs. ${ }^{8}$

Poly (ADP-ribose) polymerase 1 (PARP)-1, a constitutively expressed nuclear enzyme, belongs to a family of 18

This work was supported by National Natural Science foundation of China grants 81370263 (D.H.), 81570405 (K.H.), 81170239 (K.H.), 81270937 (K.H.), and 81300100 (Y.W.).

Y.Z., C.W., and Y.T. contributed equally to this work.

Disclosures: None declared. 
identified genes that transcribe PARPs. ${ }^{9}$ It can be activated by DNA strand breaks attributable to reactive oxygen species, which is generated at high levels under oxidative stress, thus leading to cellular damage and stimulating proinflammatory mediator production. ${ }^{10}$ In an LPS-induced endotoxic shock model, PARPI knockout (PKO) mice are strongly resistant to LPS. ${ }^{11,12}$ Along with the increased survival compared with wild-type mice, PKO mice had a lower tumor necrosis factor (TNF)- $\alpha$ and nitric oxide production with impaired transcriptional activity of NF- $\kappa \mathrm{B} .{ }^{13,14}$ In addition, PARP1 inhibition has been reported to be protective for atherosclerosis, ${ }^{15}$ $\mathrm{CCl}_{4}$-induced liver injury, ${ }^{16,17}$ ischemia-reperfusion injury, and various forms of inflammatory diseases. ${ }^{18}$ Previous reports have found significant increases in PARP activity in cultured hepatocytes under ethanol exposure or liver homogenates from rat under long-term ethanol intake. ${ }^{19,20}$ In addition, Mukhopadhyay et $\mathrm{al}^{17}$ observed a profound increase in PARP activity in hepatic tissues obtained from individuals with alcoholic cirrhosis. However, whether PARP1 plays a role in the early stage of ALD and the potential mechanism involved remains unknown.

In the present study, we hypothesized that PARP1 participates in the progression of ALD by facilitating endotoxin induced inflammatory responses. We found that PARP inhibition or genetic deletion of PARPI alleviated liver injury and lipid accumulation in long-term ethanol-fed mice. These protective effects of PARP inhibition are mediated, at least in part, by inhibiting activation of KCs.

\section{Materials and Methods}

\section{Ethics Statement}

All animal experimentation was performed in adherence with the Guide for the Care and Use of Laboratory Animals published by the US National Institutes of Health and approved by the Institutional Animal Care and Use Committee at Tongji Medical College, Huazhong University of Science and Technology.

\section{Animals and Treatment}

PKO mice (SV129 background) were purchased from Jackson Laboratory. Wild-type (WT) SV129 mice and PKO mice were maintained under standard light conditions (12-hour light, 12-hour dark cycle) at a controlled temperature (mean $\pm \mathrm{SEM}, 23^{\circ} \mathrm{C} \pm 2^{\circ} \mathrm{C}$ ) with free access to water and food. All male mice (8- to 10-week-old) were fed a control LieberdeCarli liquid diet (TROPHIC Animal Feed, High-Tech Co. Ltd., Nantong, China) for the first 3 days to acclimate to the liquid diet. Then, mice were randomly divided into two groups: ethanol-fed or pair-fed groups. Mice in the ethanolfed group were given the diet with elevating concentrations of ethanol as follows: $1.91 \%$ (v/v) and 3.83\% each for 7 days, then $5.74 \%$ for a further 2 weeks. Mice in the pair-fed group were treated with the control liquid diet containing same calories during the entire feeding period. For the pharmacologic inhibition experiments, the PARP inhibitor N-(6-oxo5,6-dihydrophenanthridin-2-yl)-2-(N,N-dimethyno)tamide (PJ34; 15 mg/kg/d, Enzo Life Sciences, Farmingdale, NY), or vehicle was administrated i.p. during the last 2 weeks before sacrifice. In the in vivo study, PJ34 was dissolved in sterile phosphate-buffered saline. Mice were sacrificed 12 hours after last injection or at the end of all experiments. Livers were weighed and excised, and randomly selected portions were snap frozen in liquid nitrogen for protein and RNA extraction or fixed in $4 \%$ paraformaldehyde for histopathologic analysis.

\section{Biochemical Analysis}

Blood samples were collected into Vacutainer hematology tubes containing 3\% EDTA (BD Biosciences, Franklin Lakes, NJ). Serum alanine aminotransferase (ALT) levels were measured using a commercially available reagent as previously described. ${ }^{21}$ Measurement of liver lipid content was performed as previously described. ${ }^{22}$ Briefly, $50 \mathrm{mg}$ of liver tissue was weighed and homogenized with $1 \mathrm{~mL}$ of buffer containing $18 \mathrm{mmol} / \mathrm{L}$ Tris, $\mathrm{pH} 7.5,300 \mathrm{mmol} / \mathrm{L}$ mannitol, $50 \mathrm{mmol} / \mathrm{L}$ EGTA, and $0.1 \mathrm{mmol} / \mathrm{L}$ phenylmethysulfonyl fluoride. A total of $400 \mu \mathrm{L}$ of the above-mentioned homogenate was mixed with $4 \mathrm{~mL}$ of chloroform/methanol (2:1) and incubated at room temperature with continuous shaking overnight. Then $800 \mu \mathrm{L}$ of $\mathrm{H}_{2} \mathrm{O}$ was added, vortexed, and centrifuged at $3000 \times g$ for 5 minutes, and then the lower lipid phase was collected and dried under nitrogen gas. The lipid pellets were dissolved with a mixture of $60 \mu \mathrm{L}$ of tert-butyl alcohol and $40 \mu \mathrm{L}$ of Triton X-114/methanol (2:1) mixture. The concentration of ALT, triglyceride, and cholesterol were then measured using assay kits (Stanbio Laboratory, Boerne, TX).

\section{Western Blot Assays}

Western blot assays were performed as described previously. ${ }^{16}$ After denaturation and SDS-PAGE, proteins were transferred to nitrocellulose membranes. Membranes were then blocked with $5 \%$ nonfat milk in $50 \mathrm{mmol} / \mathrm{L}$ Tris- $\mathrm{HCl}(\mathrm{pH}$ 7.6), $150 \mathrm{mmol} / \mathrm{L} \mathrm{NaCl}$, and $0.2 \%$ Tween 20 (TBST) for at least 2 hours. After incubation with primary antibodies [antiPoly(ADP-ribose) (PAR), Santa Cruz Biotechnology, Dallas, TX; anti-nitric oxide synthase (NOS)-2, Abcam, Cambridge, UK; anti-p65, Enzo Life Sciences; anti-PARP2, Enzo Life Sciences; anti- $\beta$-actin, Santa Cruz Biotechnology; antiglyceraldehyde-3-phosphate dehydrogenase, Santa Cruz Biotechnology; anti-Histone $\mathrm{H} 3$, Abcam] in an antibody diluent (Beyotime, Wuhan, China) at $4^{\circ} \mathrm{C}$ overnight, membranes were washed 3 times with TBST and were incubated with a peroxidase-conjugated secondary antibody in an antibody diluent at room temperature for at least 2 hours. The specific bands were detected by electrochemoluminscence detection reagents (Pierce ECL Western Blotting Substrate, Thermo Fisher Scientific, Waltham, MA). The intensity of 
the $\beta$-actin, glyceraldehyde-3-phosphate dehydrogenase, or Histone $\mathrm{H} 3$ band was used as a loading control for comparison of samples.

\section{Histopathologic Analysis}

Fixed tissues were paraffin-embedded, sectioned, and stained with hematoxylin and eosin. Frozen sections were prepared from frozen liver tissue embedded with OCT media and stained with Oil Red O. Paraffin-embedded liver sections were deparaffinized and stained for anti-PAR (Santa Cruz Biotechnology, Dallas, TX), TNF- $\alpha$ (R\&D Systems, Minneapolis, MN), cleaved-caspase-3 (Proteintech, Wuhan, China), CD68 (Boster, Wuhan, China), LY6G (Abcam), and myeloperoxidase (MPO) (Abcam). Apoptosis was detected using a TdT-mediated dUTP nick-end labeling (TUNEL) assay kit (Promega, Madison, WI) as described by manufacturer's protocol. Digital images were obtained at highpower lens by microscopy (Olympus, Tokyo, Japan). All images presented in the results are representative of at least 3 images per mouse and at least 4 mice per group.

\section{MPO Activity}

The extent of neutrophil infiltration in the liver tissues was measured by assaying MPO activity. Liver samples were harvested and stored at $-70^{\circ} \mathrm{C}$ until required for assay. MPO activity was determined by a previously described colorimetric method. ${ }^{23}$ Briefly, samples were homogenized in $50 \mathrm{mmol} / \mathrm{L} \mathrm{NaPO}_{4}$ buffer, pH 5.4, containing $0.5 \%$ hexadecyltrimethyl ammonium bromide. The homogenate was then frozen, thawed three times, and centrifuged again for 15 minutes at $4^{\circ} \mathrm{C}$. After centrifugation, supernatants were collected and mixed with tetramethylbenzidine (Sigma-Aldrich, St. Louis, MO) and $\mathrm{H}_{2} \mathrm{O}_{2}$ in phosphate buffer. The activity of MPO was assayed by measuring the change in absorbance at $450 \mathrm{~nm}$ using a microplate reader. Results are reported as units of MPO per gram of tissue.

\section{Cell Culture and Treatment}

Raw264.7 macrophages were cultured in Dulbecco's modified Eagle's medium (Gibco, Grand Island, NY) supplemented with $10 \%$ fetal bovine serum (Hyclone, Logan, UT). Cells were pretreated with PARP inhibitor PJ34 (10 $\mu \mathrm{mol} / \mathrm{L}$ ) or vehicle for 24 hours and stimulated by LPS (1 $\mathrm{ng} / \mathrm{mL}$; Sigma-Aldrich) or not for an additional 24 hours. Conditioned medium was obtained from control Raw264.7 cells (Con CM), LPS-stimulated Raw264.7 cells (LPS CM), and LPS-stimulated PJ34 pretreated Raw264.7 cells (PJ34+LPS CM) and prepared for further use. Hepatocyte AML-12 cells were cultured in Dulbecco's modified Eagle's medium/F12 supplemented with $10 \%$ fetal bovine serum, 40 $\mathrm{ng} / \mathrm{mL}$ of dexamethasone, $5 \mu \mathrm{g} / \mathrm{mL}$ of insulin, $5 \mu \mathrm{g} / \mathrm{mL}$ of transferrin, of $5 \mathrm{ng} / \mathrm{mL}$ selenium. Cells were serum starved for 24 hours and followed by treatment with $50 \mathrm{mmol} / \mathrm{L}$ ethanol for another 24 hours. In some experiments, the conditioned media from Con CM, LPS CM, or PJ34+LPS CM was applied to AML-12 hepatocytes for an additional 24 hours. Then, Oil Red O staining and TUNEL staining were performed for these special treated cells. Oil Red O staining area and TUNEL-positive cell counts were determined by computerized planimetry using Image-Pro Plus software version 6.0 (Media Cybernetics, Houston, TX). In the present in vitro study, PJ34 was dissolved in dimethylsulfoxide (DMSO; Sigma-Aldrich).

\section{Real-Time PCR}

Total RNA was purified from frozen liver tissues using Trizol (Takara Biotechnology, Otsu, Japan), and cDNA was prepared using a Reverse Transcription System kit (Takara Biotechnology) according to the manufacturer's guidelines. mRNA levels of target genes were quantified using SYBR Green Master Mix (Takara Biotechnology) with StepOne Real-Time PCR Systems (Applied Biosystems, Foster City, CA). The relative target gene expression (normalized to $\beta$-actin levels) level was calculated using the comparative threshold cycle method formula $2^{-\Delta \Delta \mathrm{Ct}}$. Primer sequences were given in Table 1.

\section{Electromobility Shift Assays}

DNA combination activation of NF- $\kappa \mathrm{B}$ was detected using Light Shift Chemiluminescent Electromobility Shift Assay kit (Thermo Fisher Scientific) according to the manufacturer's protocol. The oligonucleotides sequences of NF- $\mathrm{BB}$ combination element were forward: 5'-AGTTGAGGGGACTTTCCCAGGC-3'; and reverse: 5'-GCCTGGGAAAGTCCCCTCAACT- $3^{\prime}$. Biotin was labeled at the $5^{\prime}$ end of the forward oligonucleotides. In brief, binding reactions containing $5 \mu \mathrm{g}$ of nuclear extracts and $50 \mathrm{fM}$ of oligonucleotide were performed for 30 minutes at room temperature. Then, protein-DNA complexes were subjected to $6 \%$ native polyacrylamide gel electrophoresis and transferred to nylon membranes, which were immediately cross-linked on a UV crosslinker. Bands were then detected by using the chemiluminescent method.

\section{Statistical Analysis}

All the values are means \pm SEM. Statistical analysis was performed by one-way analysis of variance, followed by Student-Newman-Keuls multiple comparison tests. The analysis was conducted using SPSS software version 11.0 (SPSS Inc., Chicago, IL). Unless otherwise stated, the level of significance was 0.05 .

\section{Results}

\section{Ethanol Consumption Induces PARP Activation in Mouse Liver}

Previous studies have found that ethanol exposure resulted in a significant increase in PARP activity in cultured 
Table 1 Mouse Primers Used for Real-Time RT-PCR

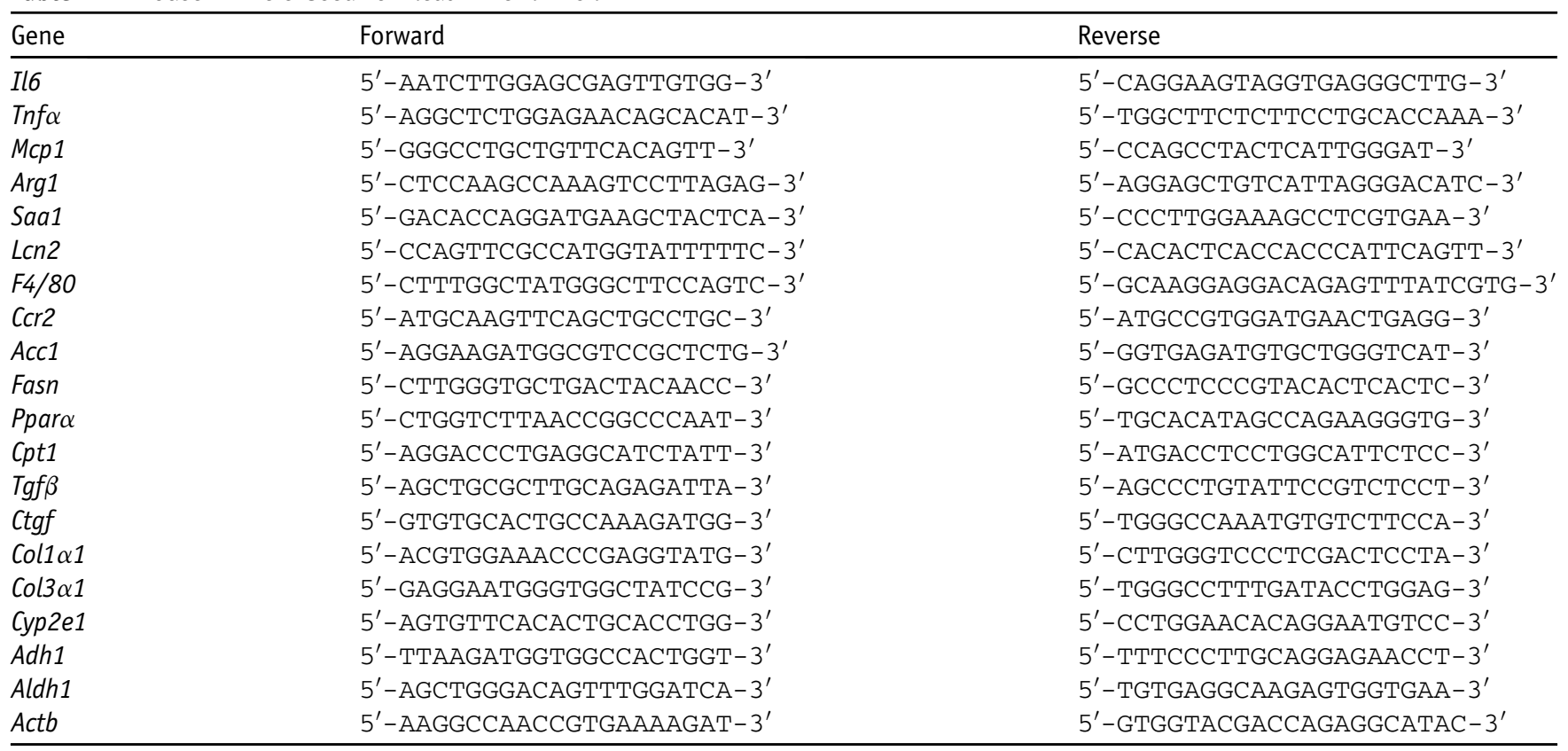

hepatocytes or liver homogenates. ${ }^{19,20}$ In the present study, 129S1/SvImJ mice received a liquid ethanol diet for 4 weeks totally. For the pharmacologic inhibition experiments, mice were injected with PARP inhibitor PJ34 (15 mg/kg/d) during the last 2 weeks. We performed Western blot and immunohistochemistry by using anti-PAR antibody, which is specific for PAR polymers 2 to 50 units long, to assess poly(ADP-ribosyl)ation (PARylation) levels in liver samples totally or in situ. Our results revealed that this kind of posttranscriptional modification was detectable at basal conditions, and the hepatic PARylation levels were markedly elevated after 4-week ethanol feeding (Figure 1, A and B). Treatment with PARP inhibitor PJ34 efficiently inhibited the ethanol-induced increases in PARylation levels (Figure 1, A and B). These results indicated that 4-week ethanol feeding induced PARP activation in liver of mice. Moreover, a single dosage (5-g/kg gavage) of ethanol administration could also stimulate profound PARylation in mouse liver (Figure 1C). All these results suggested that PARP activation was involved in the progression of ALD.

\section{Pharmacologic Inhibition of PARP Alleviates Long-Term Ethanol-Induced Liver Steatosis}

Long-term ethanol feeding to mice results in the development of hepatic steatosis and inflammatory cytokine expression. ${ }^{6,8,24}$ To investigate the effect of PARP inhibition on the progression of ALD, levels of hepatic neutral fat were visualized by Oil Red $\mathrm{O}$ staining, followed by extraction and quantification of triglycerides and cholesterol contents. Oil Red O staining of liver sections and enzymatic colorimetric assay of extracted lipids revealed that ethanol feeding for 4 weeks resulted in excessive lipid accumulation and increased triglycerides levels in the liver of 129S1/ SvImJ mice (Figure 2, A and B). We also found that mice fed with ethanol had increased serum ALT levels and liver weight to body weight (LW/BW) ratio (Table 2). These results indicated that long-term ethanol feeding led to alcoholic liver injury and progressed to liver steatosis. However, no significant changes were observed in hepatic cholesterol content between ethanol-fed mice treated with PJ34 or not (Figure 2C). Mice treated with PARP inhibitor PJ34 had marked reduction in liver content of triglycerides (Figure 2, A and B) and significant decreases in serum ALT levels compared with ethanol-fed mice (Table 2). Ethanol consumption levels of mice treated with PJ34 or vehicle were comparable (Table 2).

We further performed real-time RT-PCR to explore the effects of PARP inhibition on the expression of genes involved in hepatic lipid metabolism and ethanol metabolism. As expected, mice fed with ethanol had increased hepatic expression of lipogenic genes, including acetyl-CoA carboxylase-1 (Accl) and fatty acid synthase (Fasn) (Figure 2D), and decreased expression of lipolysis genes, including Ppar $\alpha$ and Cptl (Figure 2E). Treatment with PJ34 effectively reduced hepatic expression of genes involved in lipogenesis (Figure 2D) and elevated hepatic expression of genes involved in lipolysis (Figure 2E). However, no significant changes were observed in the expression of ethanol metabolism-related genes, including Cyp2e1, Adh1, and Aldh1 (Supplemental Figure S1A). In addition, we found profound hepatic fibrosis and increased expression of fibrogenic genes (including $\operatorname{Col} 1 \alpha 1$ and $\operatorname{Col} 3 \alpha 1$ ) in ethanolfed mice (Figure 2, A, F and G). However, no significant attenuation was detected in PJ34-treated mice (Figure 2, A, $\mathrm{F}$, and $\mathrm{G})$. These results indicated that PARP activation 

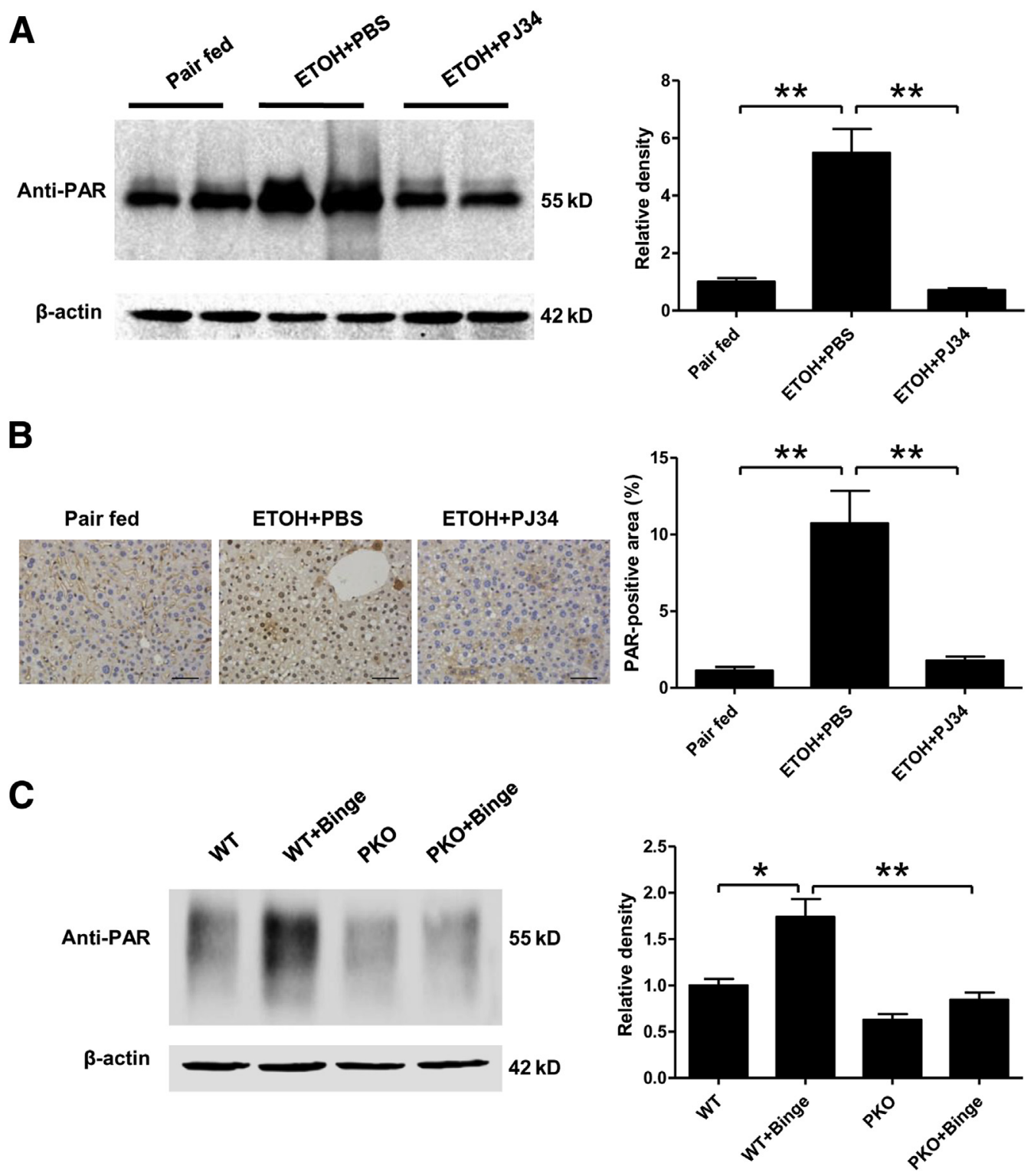

Figure 1 Ethanol (ETOH) consumption increases poly(ADP-ribosyl)ation (PAR) levels in mouse liver. A: PAR levels in the indicated groups were shown in the left panel and quantified in the right panel. B: Immunohistochemical staining of poly(ADP-ribosyl)ated proteins in liver tissue from mice in the indicated groups. PAR-positive area was calculated in the right panel. C: Wild-type (WT) and PARP1 knockout (PKO) mice exposed to a single dosage of ETOH (5 $\mathrm{g} / \mathrm{kg}$ ). Poly(ADP-ribosyl)ation levels in the indicated groups were shown in the left panel and quantified in the right panel. $n=4$ (A-C); Original magnification, $40 \times$ objective (scale bar $=50 \mu \mathrm{m}(\mathrm{B}) .{ }^{*} P<0.05,{ }^{*}{ }^{*} P<0.01 . \mathrm{PJ} 34, \mathrm{~N}-(6-0 \times 0-5,6$-dihydrophenanthridin-2-yl)-2-(N,N-dimethyno)tamide.

contributed to the progression of ethanol-induced liver steatosis by modulating lipid metabolism relevant genes expression.

\section{Genetic Depletion of PARP1 Protects Long-Term Ethanol-Induced Liver Steatosis}

Because PARP1 is the most abundant member of the PARP family and accounts for approximately $90 \%$ of cellular PARP activity, we used PKO male mice to perform a genetic depletion assay. PKO mice and their WT littermates were fed with ethanol for 4 weeks. Compared with WT counterparts, PKO mice receiving ethanol feeding had markedly lowered hepatic PARylation levels (Figure 3A), confirming that hepatic PARP1 is the primary contributor of enhanced PARylation. In addition, we found no significant change in PARP2 protein levels in ethanol-fed mice and no compensatory increase in pair-fed or ethanol-fed PKO mice (Supplemental Figure S2, A and B).

Similarly, we found significantly decreased serum ALT values and LW/BW ratios in ethanol-fed PKO mice compared with WT littermates (Table 3). Ethanol consumption levels and hepatic expression of enzymes metabolizing ethanol in these two mice strains were identical (Table 3 and Supplemental Figure S1B). PKO 


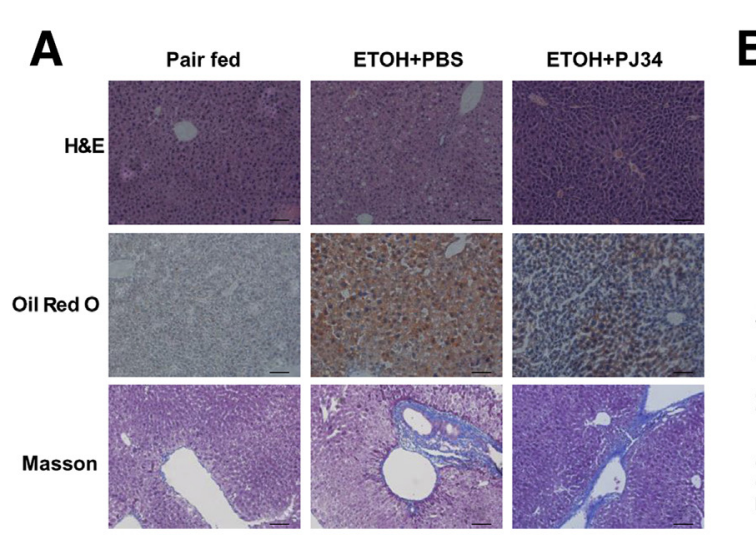

B

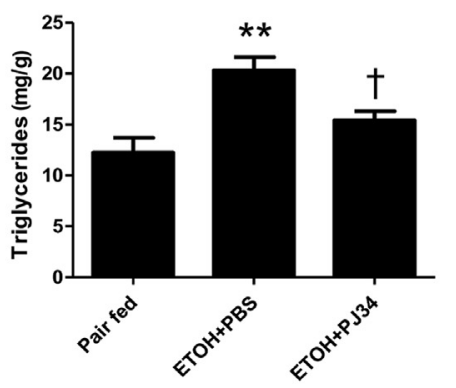

C

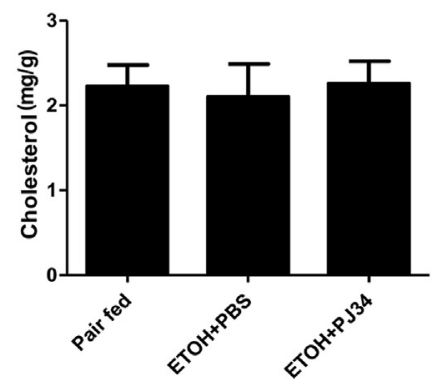

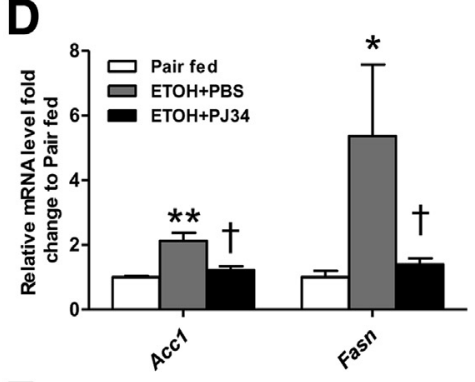

F

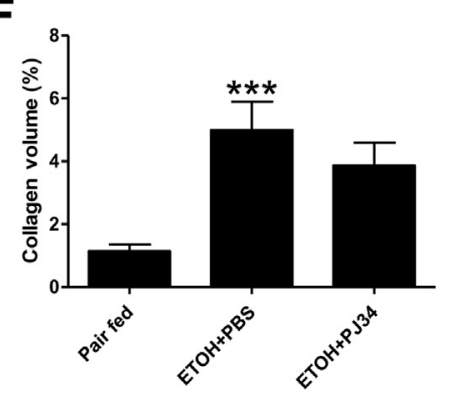

E

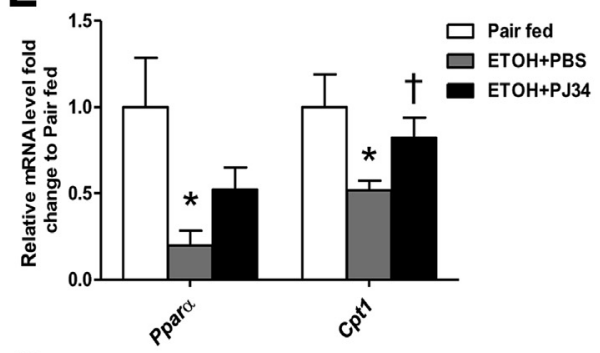

G

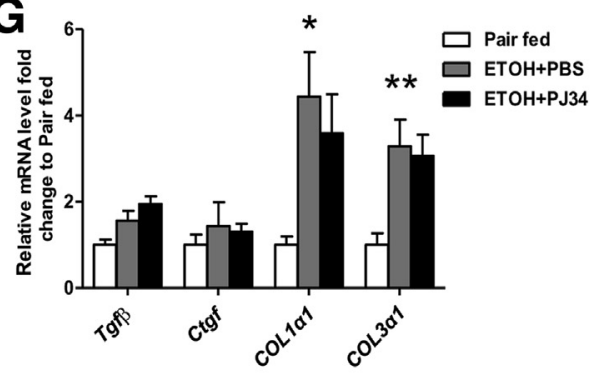

Figure 2 Poly (ADP-ribose) polymerase (PARP) inhibitor alleviates chronic alcoholic liver steatosis. A: Hematoxylin and eosin (H\&E), Oil Red 0, and Masson trichrome staining of liver sections from the indicated groups are shown. Hepatic triglycerides contents (B) and hepatic cholesterol contents $(\mathbf{C})$ were measured by biochemical assays. D and E: Hepatic mRNA levels of Acc1, Fasn, Ppard, and Cpt1 in the indicated groups were measured by real-time RT-PCR. F and G: Hepatic mRNA levels of $\operatorname{Tgf} \beta, \operatorname{Ctgf}, \operatorname{Col} 1 \alpha 1$, and $\operatorname{Col} 3 \alpha 1$ in the indicated groups were measured by real-time RT-PCR. $n=5$ for the pair-fed group and 6 for the ethanol and phosphate-buffered saline (ETOH+PBS) and ethanol and $\mathrm{N}$-(6-oxo-5,6-dihydrophenanthridin-2-yl)2-(N,N-dimethyno)tamide (ETOH +PJ34) groups $(\mathbf{B}-\mathbf{G})$. Scale bar $=100 \mu \mathrm{m}(\mathbf{A})$. Original magnification: $\times 20(\mathbf{A}) .{ }^{*} P<0.05,{ }^{*} P P<0.01$, and $* * * P<0.001$ versus the pair-fed group; ${ }^{\dagger} P<0.05$ versus the $\mathrm{ETOH}+\mathrm{PBS}$ group.

mice also had significant decreases in hepatic lipid deposition and triglycerides levels compared with ethanol-fed WT littermates (Figure 3, B and C). However, no significant changes were observed in hepatic cholesterol content between WT and PKO mice (Figure 3D). Consistent with these results, depletion of PARP1 resulted in decreased expression of lipogenic genes (Accl and Fasn) (Figure 3E) and increased expression of lipolysis genes
(Ppara and Cptl) (Figure 3F). In addition, we found profound hepatic fibrosis and increased fibrogenic gene expression in ethanol-fed mice (Figure 3, B, G, and H). However, no significant attenuation was detected in ethanol-fed PKO mice (Figure 3, B, G, and H). Collectively, these results indicated that increased PARP activity, mainly mediated by PARP1, aggravated hepatic steatosis in long-term alcohol-feeding mice.

Table 2 General Characteristics of Mice in Pharmacologic Inhibition Experiments

\begin{tabular}{lccc}
\hline Parameter & Pair fed $(n=5)$ & ETOH+PBS $(n=6)$ & ETOH+PJ34 $(n=6)$ \\
\hline Body weight before alcohol $(\mathrm{g})$ & $22.8 \pm 0.8$ & $23.2 \pm 0.6$ & $23.5 \pm 1.0$ \\
Body weight 2 weeks after alcohol (g) & $23.4 \pm 0.8$ & $22.9 \pm 0.7$ & $23.9 \pm 0.4$ \\
Body weight 4 weeks after alcohol (g) & $24.0 \pm 0.9$ & $21.0 \pm 0.6^{*}$ & $21.4 \pm 0.5^{*}$ \\
Alcohol consumption (g/kg/day) & & $47.4 \pm 1.7$ & $49.7 \pm 2.8$ \\
Liver weight/Body weight (\%) & $3.6 \pm 0.1$ & $4.3 \pm 0.2^{*}$ & $4.1 \pm 0.2$ \\
ALT (IU/L) & $31.5 \pm 3.9$ & $74.6 \pm 5.0^{* *}$ & $48.4 \pm 4.0^{* \dagger}$ \\
\hline
\end{tabular}

Data are expressed as means \pm SEM.

${ }^{*} P<0.05,{ }^{* *} P<0.01$ versus the pair-fed group; ${ }^{\dagger} P<0.05$ versus the $\mathrm{ETOH}+\mathrm{PBS}$ group.

ALT, alanine aminotransferase; ETOH, ethanol; PBS, phosphate-buffered saline. 
A

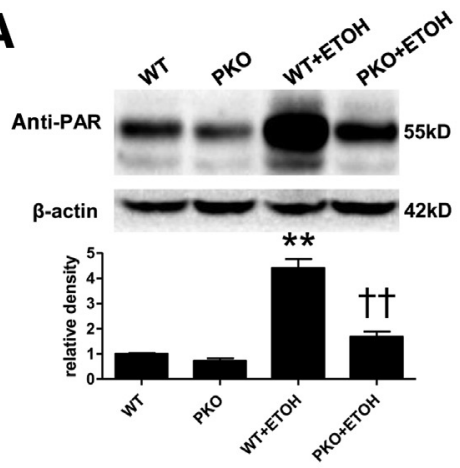

C

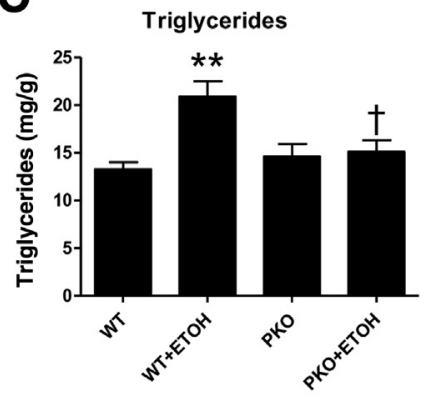

E

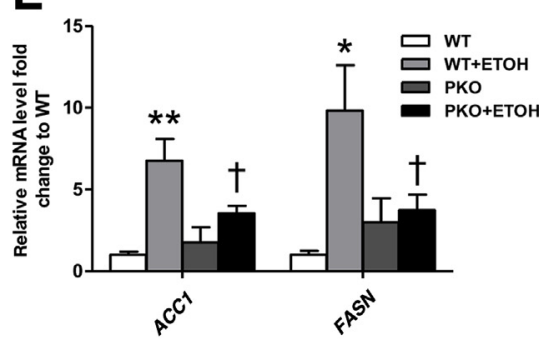

G

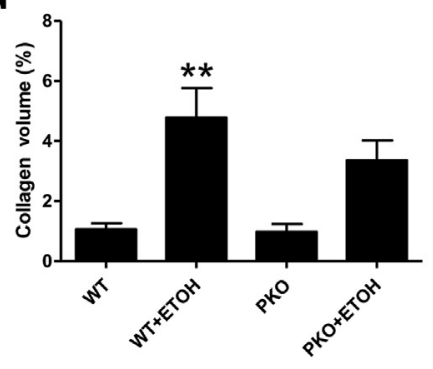

B

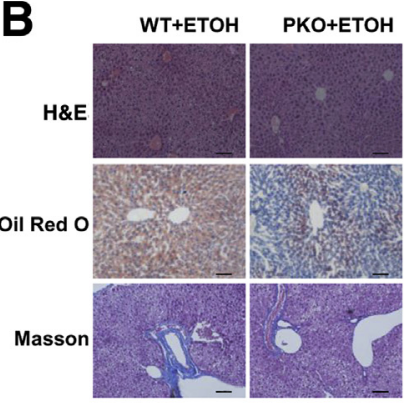

D

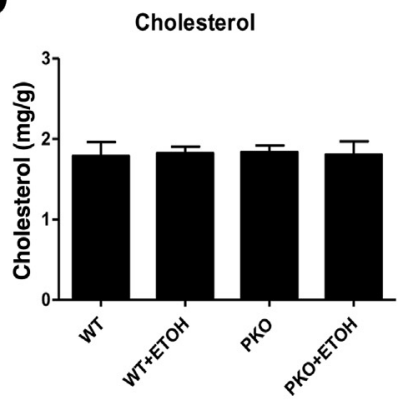

F

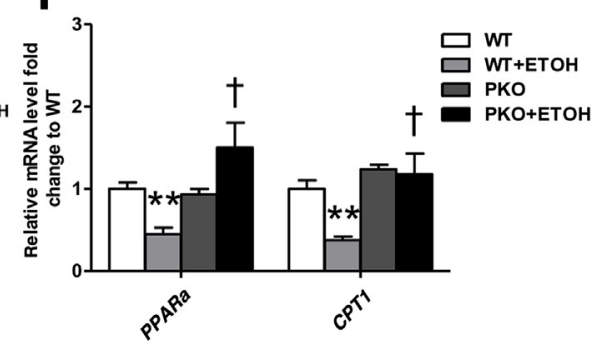

H

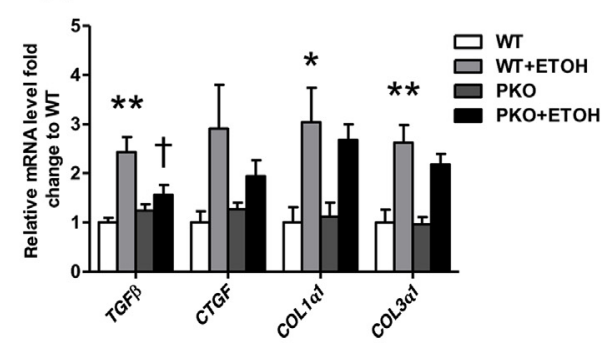

Figure 3 Genetic deletion of poly (ADP-ribose) polymerase (PARP)-1 protects chronic alcoholic liver steatosis. Wild-type (WT) mice or PARP1 knockout (PKO) mice were treated with ethanolcontaining diets or pair-fed control diets for 28 days. A: Poly(ADP-ribosyl)ation levels in the indicated groups were shown in the upper panel and quantified in the lower panel. B: Hematoxylin and eosin (H\&E), Oil Red 0, and Masson trichrome staining of liver sections from the indicated groups are shown. Hepatic triglycerides contents (C) and cholesterol contents (D) in the indicated groups were measured by biochemical assays. $\mathbf{E}$ and $\mathbf{F}$ : Hepatic mRNA levels of Acc1, Fasn, Ppar $\alpha$, and Cpt1 in the indicated groups were measured by real-time RT-PCR. $\mathbf{G}$ and $\mathbf{H}$ : Hepatic mRNA levels of $\operatorname{Tgf} \beta, \operatorname{Ctgf}, \operatorname{Col} 1 \alpha 1$, and $\operatorname{Col} 3 \alpha 1$ in the indicated groups were measured by real-time RT-PCR. $n=4$ (A); $n=6$ for each group $(\mathbf{C}-\mathbf{H})$. Scale bar $=100$ $\mu \mathrm{m} \quad$ (B). Original magnification: $\times 20$ (B). ${ }^{*} P<0.05,{ }^{*} P<0.01$ versus the pair-fed group; ${ }^{\dagger} P<0.05,{ }^{\dagger \dagger} P<0.01$ versus the wild-type and ethanol (WT+ETOH) group.
Inhibiting PARP1 Limits KC Activation and Neutrophilic Infiltration Induced by Ethanol Consumption in Vivo

Activation of KCs plays a central role in the progression of ALD, and limiting their polarization into a M1 phenotype is proved to be an effective therapeutic strategy in ALD animal model. ${ }^{6,8}$ Thus, we next investigated whether PARP1 regulates $\mathrm{KC}$ activation in the progression of ALD in vivo. We found that long-term ethanol consumption increased hepatic expression of
M1 markers (Tnf $\alpha$, Il6, Monocyte chemotactic protein-1, and Nos-2) compared with pair-fed controls (Figure 4, A and B). The elevated mRNA levels of M1 genes were significantly reversed by $\mathrm{PJ} 34$ treatment or PARP1 depletion (Figure 4, A and B), whereas the M2 marker Argl was unchanged (Figure 4, A and B). In accordance with its mRNA level, immunofluorescence staining revealed significantly decreased TNF- $\alpha$ level in the liver segments of the PJ34-treated mice or PKO mice (Figure 4C and Supplemental Figure S3A). In addition, we evaluated signs of macrophagocytes in liver samples, 
Table 3 General Characteristics of Mice in Genetic Depletion Experiments

\begin{tabular}{lcccc}
\hline Parameter & WT $(n=6)$ & WT+ETOH $(n=6)$ & PKO $(n=6)$ & PKO+ETOH $(n=6)$ \\
\hline Body weight before alcohol (g) & $22.9 \pm 0.4$ & $23.6 \pm 1.1$ & $23.0 \pm 0.6$ & $23.7 \pm 1.1$ \\
Body weight 2 weeks after alcohol (g) & $23.6 \pm 0.9$ & $23.0 \pm 1.4$ & $23.1 \pm 0.7$ & $23.4 \pm 0.9$ \\
Body weight 4 weeks after alcohol (g) & $24.9 \pm 1.5$ & $20.5 \pm 1.6^{*}$ & $23.9 \pm 1.6$ & $20.7 \pm 0.5^{*}$ \\
Alcohol consumption (g/kg/day) & & $45.2 \pm 2.7$ & $46.9 \pm 2.0$ \\
Liver weight/body weight (\%) & $3.8 \pm 0.2$ & $5.7 \pm 0.7^{* *}$ & $4.0 \pm 0.1$ & $4.5 \pm 0.2^{\dagger}$ \\
ALT (IU/L) & $29.8 \pm 2.6$ & $73.7 \pm 5.2^{* *}$ & $33.7 \pm 2.4$ & $50.2 \pm 2.5^{* \dagger}$ \\
\hline
\end{tabular}

Data are expressed as means \pm SEM.

${ }^{*} P<0.05,{ }^{* *} P<0.01$ versus $\mathrm{WT} ;{ }^{\dagger} P<0.05$ versus $\mathrm{WT}+\mathrm{ETOH}$.

ALT, alanine aminotransferase; ETOH, ethanol; PKO, PARP1 knockout; WT, wild type.

and no significant difference was found in hepatic F4/80 and $\mathrm{C}-\mathrm{C}$ chemokine receptor type 2 mRNA levels (Figure 4, A and B) and CD68-positive cells (Figure 4D and Supplemental Figure S3B). Therefore, we concluded that long-term ethanol consumption activated resident $\mathrm{KCs}$, which could be successfully reversed by PARP1 inhibition.

We further detected hepatic expression of Saal and Lcn2. mRNA levels of these two acute-phase protein genes were profoundly increased in ethanol-fed mice and attenuated in PJ34-treated mice or PKO mice (Figure 4, A and B). A recent study revealed that $L c n 2$ propagates the development of ALD through driving neutrophilic infiltration. ${ }^{25}$ Therefore, immunostaining of Ly6G and MPO and MPO activity detection were performed to assess neutrophilic infiltration. Ethanol-fed mice exhibited increased numbers of Ly6G or MPO-positive cells and elevated hepatic MPO activity compared with pair-fed mice (Figure 4, E-G). Ethanol-fed mice treated with PJ34 exhibited decreased numbers of Ly6G- or MPOpositive cells and alleviated hepatic MPO activity compared with ethanol-fed mice (Figure 4, E-G). In addition, we got similar results in our genetic depletion experiments (Supplemental Figure S3, C-E). Taken together, these results indicated that enhanced hepatic neutrophils infiltration induced by ethanol consumption was attenuated by PARP inhibition.

\section{Inhibition of PARP1 Protects Hepatocytes from Apoptosis Induced by Long-Term Ethanol Feeding}

Apoptosis is one of the hallmarks of ethanol-induced liver injury. Ethanol sensitizes hepatocytes to apoptosis both in vivo ${ }^{26}$ and in vitro. ${ }^{27,28}$ To examine whether the blockage of ethanol injury resulting from PARP1 inhibition was mediated by inhibition of cell apoptosis, immunohistochemical staining with cleaved caspase-3, a specific proapoptotic factor, was performed on these liver sections. As shown in Figure 5A, the number of cleaved caspase3-positive cells was significantly increased in ethanol-fed livers compared with pair-fed livers. As expected, increased cleaved caspase-3-positive cells induced by ethanol consumption was attenuated by PARP inhibition
(Figure 5A). In addition, TUNEL staining validated the protective effect of PJ34 on ethanol-induced hepatocyte damage (Figure 5B). Consistent with the pharmacologic inhibition results, genetic depletion of PARP1 alleviated cell apoptosis in alcoholic liver tissue (Figure 5, C and D). Moreover, no obvious difference was found between pairfed mice treated with PJ34 or vehicle (Supplemental Figure S4, A-C). Collectively, these results indicated that ethanol-fed mice had predominant apoptosis signals, which could be reduced by PJ34 treatment or PARPI gene depletion.

\section{Limiting Macrophage Activation Protects Hepatocytes} from Steatosis and Apoptosis in Vitro

To further detect the protective role of PARP inhibition, we performed a cell model to mimic the double hit that cooperatively induces hepatocyte steatosis and apoptosis. ${ }^{8}$ Neither ethanol nor LPS alone could induce AML-12 hepatocyte steatosis or apoptosis (data not shown). However, when collaborated with alcohol administration, LPS CM effectively promoted hepatocyte lipid accumulation and apoptosis (Figure 6, A and B). However, pretreatment of PJ34 could nearly reverse these detrimental effects (Figure 6, A and B). Furthermore, we found that LPS induced significant elevation of mRNA levels of $T n f \alpha, I l 6$, Monocyte chemotactic protein-1, and Nos 2 and protein levels of Nos 2 in Raw264.7 cells, which were effectively reversed by PARP1 inhibition (Figure 6, C and D). Further detection of Lcn2 revealed similar results (Supplemental Figure S5). However, no significant change was detected in mRNA levels of Saal (Supplemental Figure S5), which is mainly produced by hepatocytes in response to stress. We further performed electromobility shift assay and Western blot assay to assess nuclear translocation and DNA-binding capacity of NF- $\kappa \mathrm{B}$ in Raw264.7 cells. We found that LPS stimulation resulted in obvious nuclear translocation of p65 and enhanced DNA-binding activity of NF- $\mathrm{KB}$ (Figure 6, E and F). However, pretreatment of PJ34 successfully reversed these detrimental effects (Figure 6, E and F). Thus, these results indicate that pretreatment of PARP inhibitor PJ34 is sufficient to inhibit LPS-induced macrophages activation and subsequently 

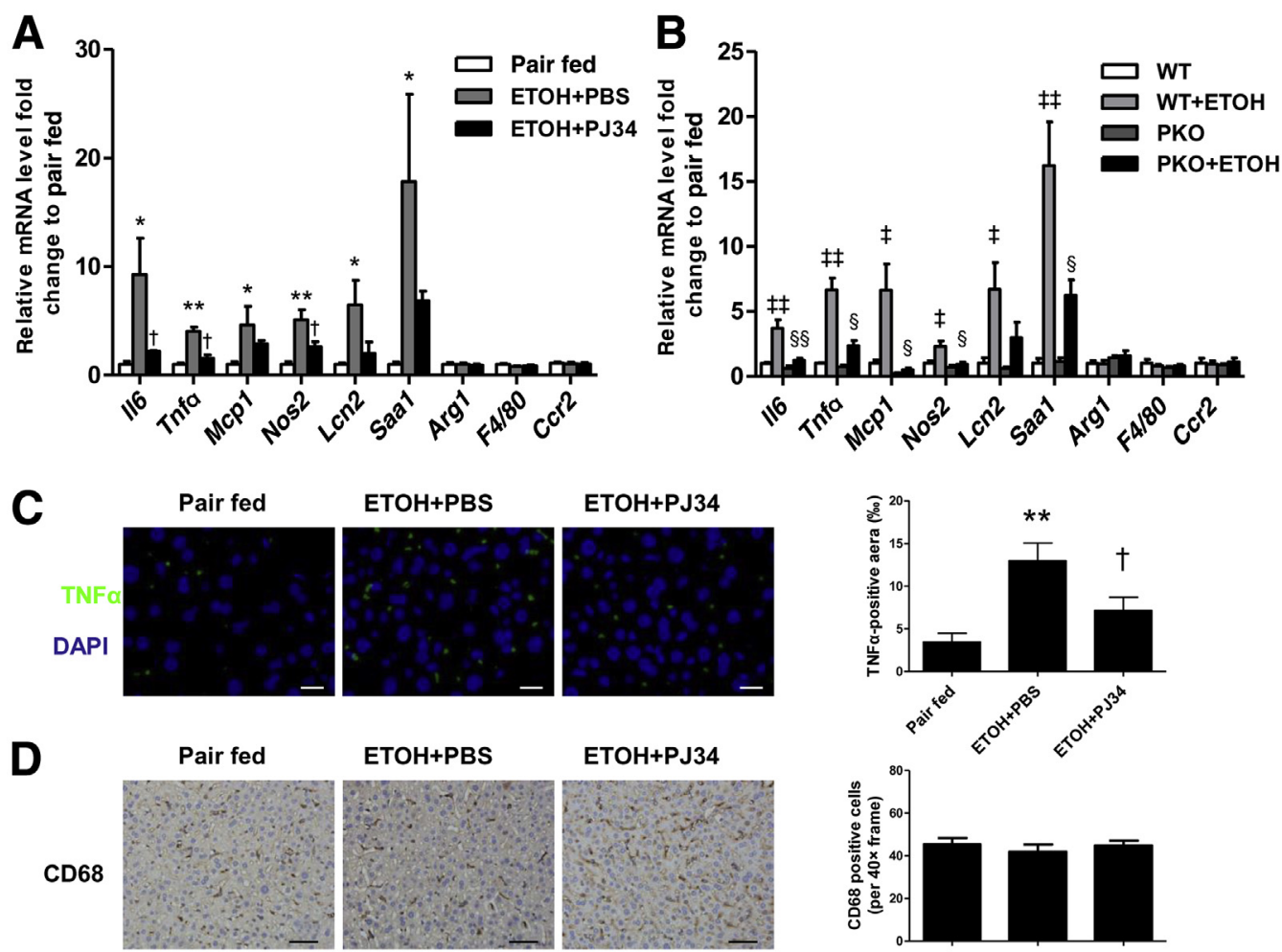

ETOH+PBS

ETOH+PJ34
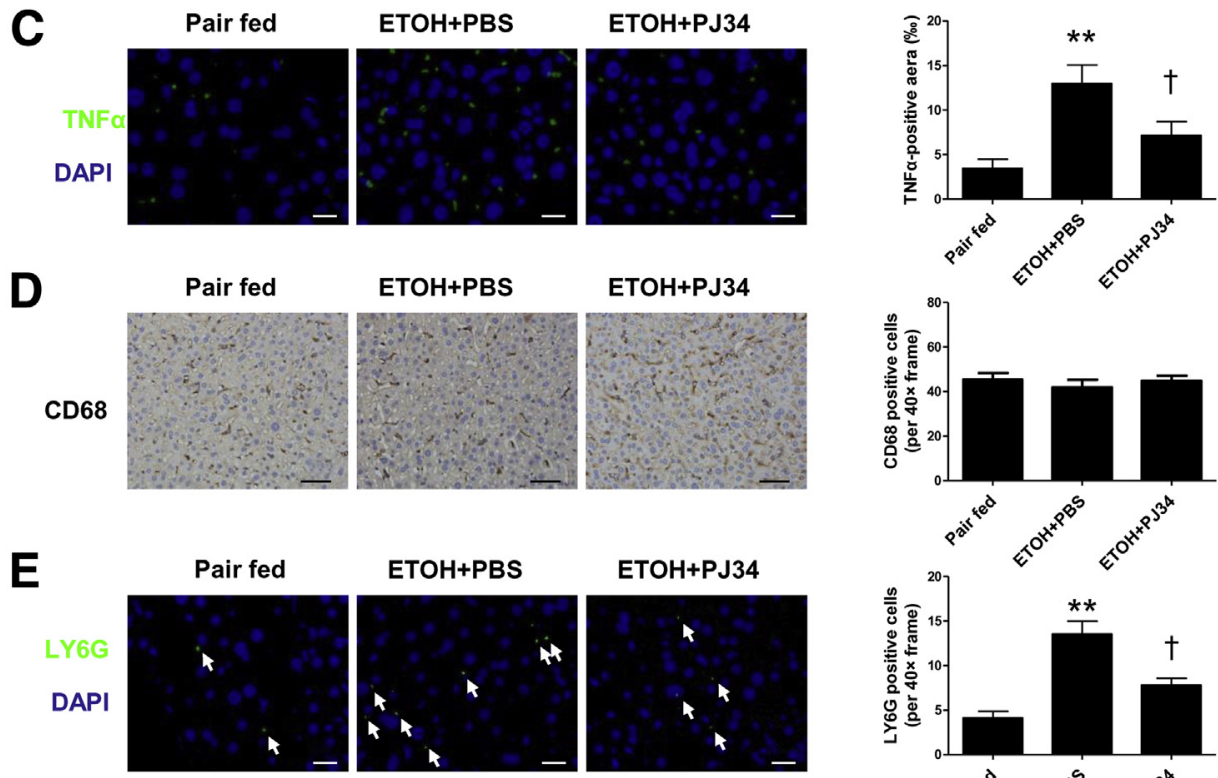

ETOH+PBS

ETOH+PJ34
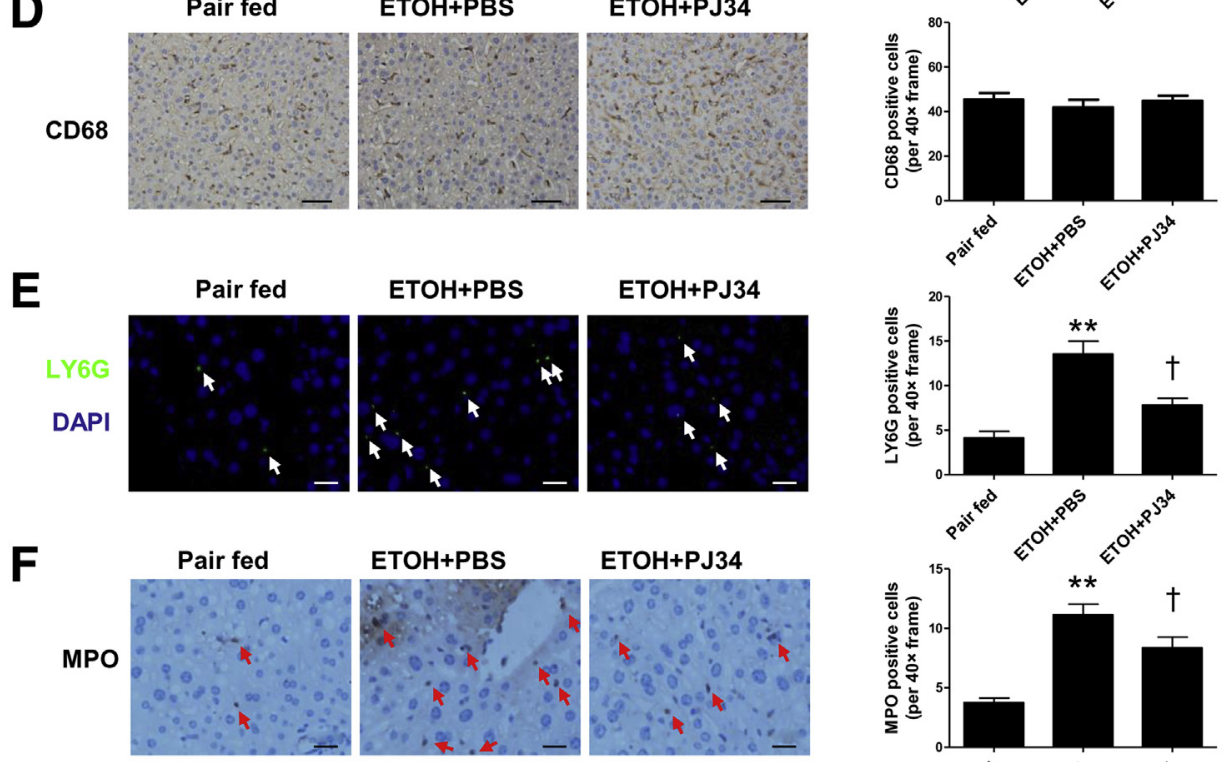

ETOH+PBS

ETOH+PJ34
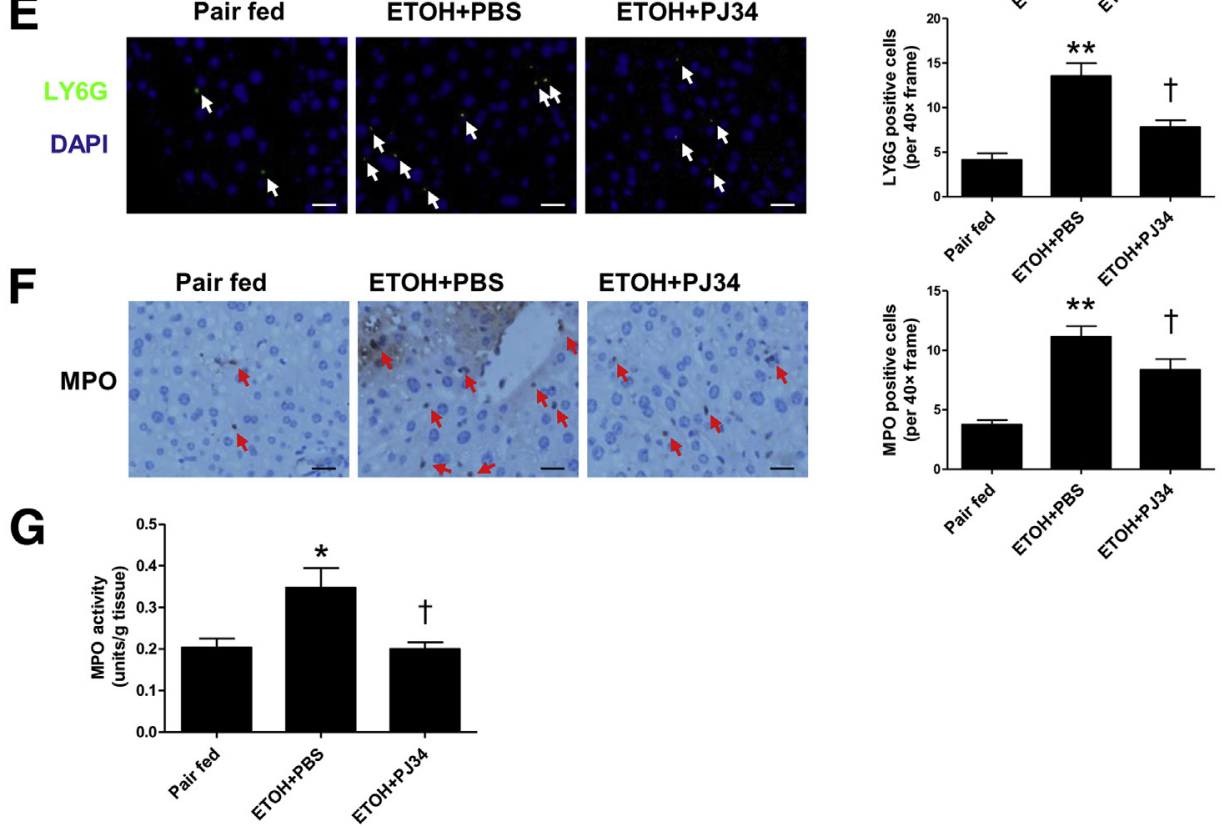

Figure 4 Poly (ADP-ribose) polymerase (PARP) inhibitor limits Kupffer cell (KC) M1 polarization and neutrophil infiltration. A: Real-time RT-PCR analysis of hepatic M1 (Il6, Tnfo, Mcp1, and Nos2) genes, M2 (Arg1) genes, acute-phase protein genes (Lcn2, Saa1), and macrophages marker (F4/80, Ccr2) genes in the indicated groups. B: Real-time RT-PCR analysis of hepatic M1 genes, M2 genes, acute-phase protein genes, and macrophages marker genes in wild-type (WT) or PARP1 knockout (PKO) mice treated with control liquid diets or ethanol (ETH0)-containing diets. C: Tumor necrosis factor- $\alpha$ protein levels were evaluated by immunostaining in paraffin-embedded liver sections. D-F: CD68-positive cells, Ly6G-positive cells, and myeloperoxidase (MP0)-positive cells were evaluated by immunostaining in paraffin-embedded liver sections. Ly6G-positive cells and MP0-positive cells are indicated by white arrows and red arrows, respectively. Positive cell counts per $40 \times$ frame were quantified and shown in the right panel, respectively. G: Hepatic MPO activity in the indicated groups was evaluated. $n=5$ for the pair-fed group $(\mathbf{A}$ and $\mathbf{C}-\mathbf{G}) ; n=6$ for the ETOH and phosphate-buffered saline (ETOH+PBS) and ETOH and N-(6-oxo-5, 6-dihydrophenanthridin2-yl)-2-(N,N-dimethyno)tamide (ETOH+PJ34) groups (A and $\mathbf{C}-\mathbf{G}) ; n=6$ for each group (B). ${ }^{*} P<0.05,{ }^{*} P<0.01$ versus the pair-fed group; ${ }^{\dagger} P<0.05$ versus the ETOH+PBS group (A and $\mathbf{C}-\mathbf{G}$ ); ${ }^{\ddagger} P<0.05,{ }^{\ddagger \ddagger} P<0.01$ versus the WT group; ${ }^{\S} P<0.05,{ }^{\S \S} P<0.01$ versus the WT+ETOH group (B). Original magnification: $\times 40(\mathbf{C}-\mathbf{F})$. Scale bars: $20 \mu \mathrm{m}(\mathbf{C}, \mathbf{E}$, and $\mathbf{F}) ; 50 \mu \mathrm{m}$ (D). 


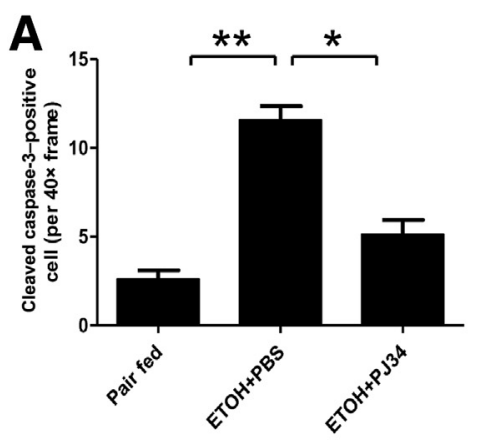

Pair fed

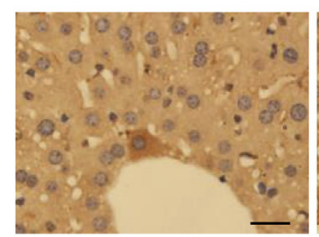

ETOH+PBS

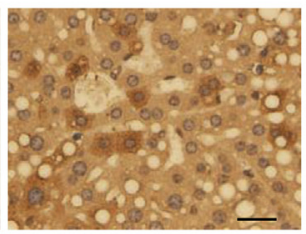

Pair fed

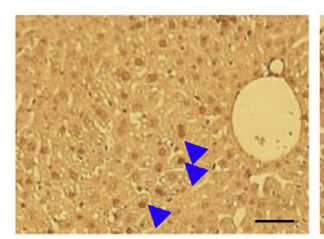

ETOH+PBS

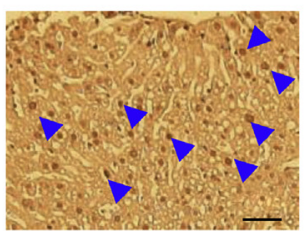

ETOH+PJ34

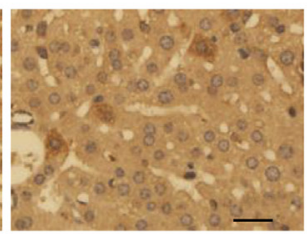

ETOH+PJ34

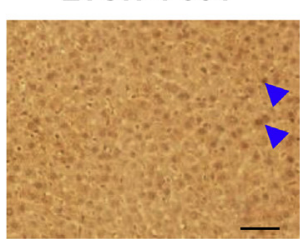

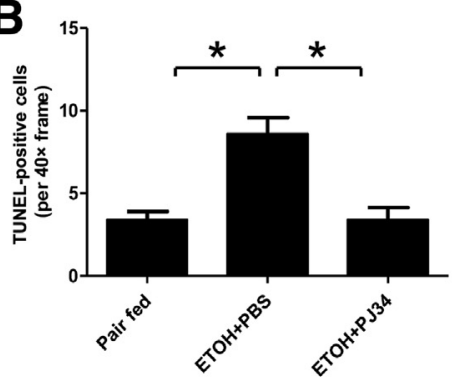

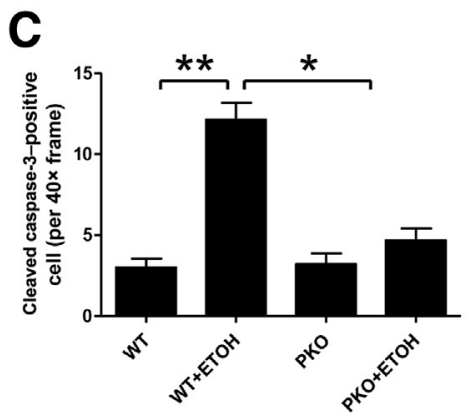

WT

WT+ETOH

PKO

PKO+ETOH
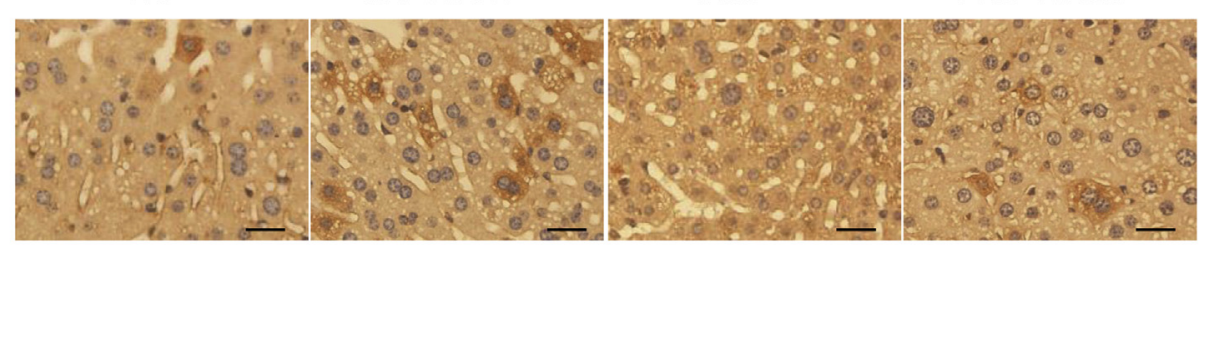

WT

WT+ETOH

PKO

PKO+ETOH
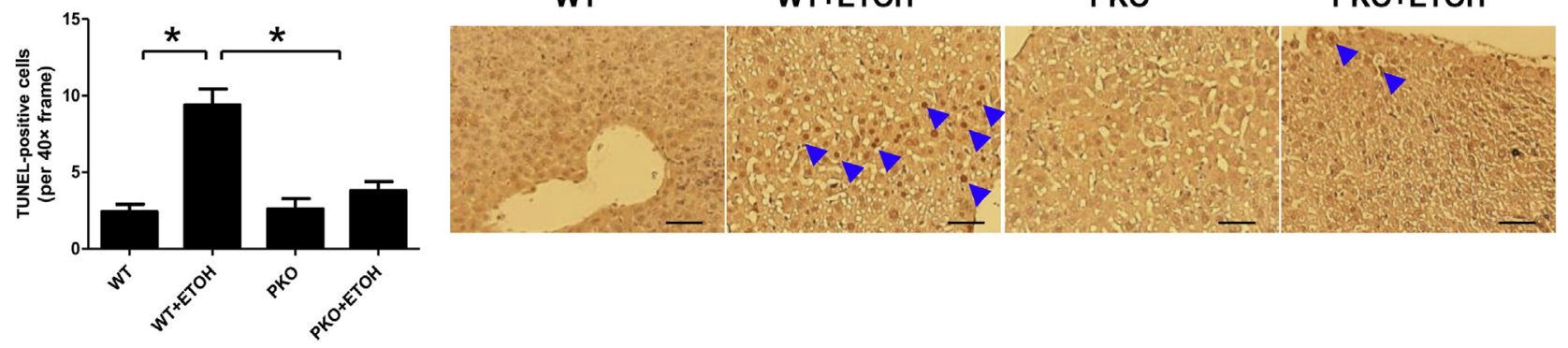

Figure 5 Inhibition of poly (ADP-ribose) polymerase (PARP)-1 reduces chronic alcoholic liver apoptosis. A and B: Immunohistochemistry toward cleaved caspase-3 or TdT-mediated dUTP nick-end labeling (TUNEL) staining was applied to formalin-fixed liver sections from mice treated with pair-fed control diets, treated with ethanol (ETOH)-containing diets, or co-treated with N-(6-oxo-5,6-dihydrophenanthridin-2-yl)-2-(N,N-dimethyno)tamide (PJ34). Apoptotic cells were counted in three independent sections. $\mathbf{C}$ and $\mathbf{D}$ : Immunohistochemistry toward cleaved caspase-3 or TUNEL staining was evaluated in paraffin-embedded liver section from wild-type (WT) and PARP1 knockout (PKO) mice treated with a control liquid diet or ETOH-containing diet. TUNEL-positive cells are indicated by arrowheads. Apoptotic cells were counted in three independent sections. $n=5$ for each group (A and $\mathbf{B}) ; n=5$ for each group (C and $\mathbf{D})$. Original magnification: $\times 40(\mathbf{A}$ and $\mathbf{C}) ; \times 20(\mathbf{B}$ and $\mathbf{D})$. Scale bars: $50 \mu \mathrm{m}(\mathbf{A}$ and $\mathbf{C}) ; 100 \mu \mathrm{m}(\mathbf{B}$ and $\mathbf{D}) .{ }^{*} P<0.05,{ }^{*} P<0.01$.

protect hepatocytes from the double hit induced injury in vitro.

\section{Discussion}

The activation of PARP1 is involved in the DNA damage response, telomere maintenance, and stress responses. ${ }^{9}$ In addition, pharmacologic PARP inhibitors recently have been shown to have significant protective effects on some stressrelated diseases, such as cardiovascular diseases, stroke, metabolic disorders, diabetes, and autoimmunity. ${ }^{29-33}$ In the present study, we found that long-term alcohol feeding resulted in PARP activation, as indicated by PARylation levels in the liver, accompanied with elevated serum ALT levels and severe hepatic steatosis. Moreover, PARP inhibition or genetic depletion of $P A R P 1$ protected the liver under 
A
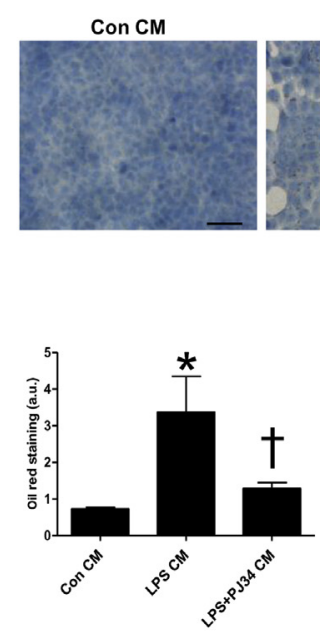

C

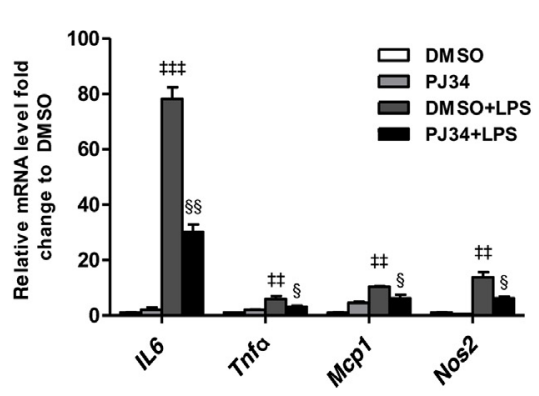

$\mathbf{E}$
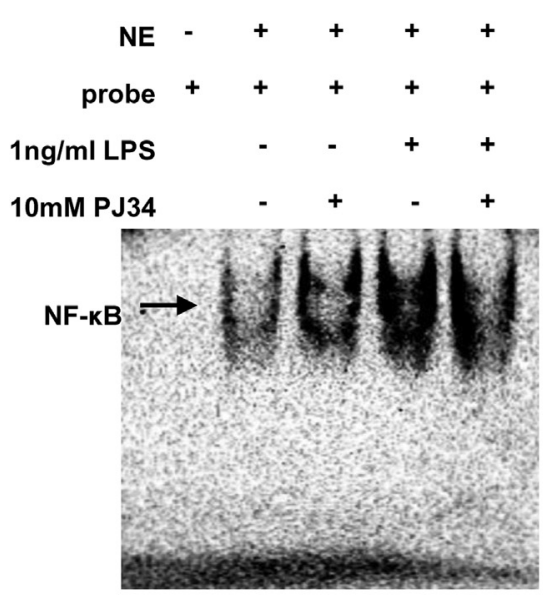

B

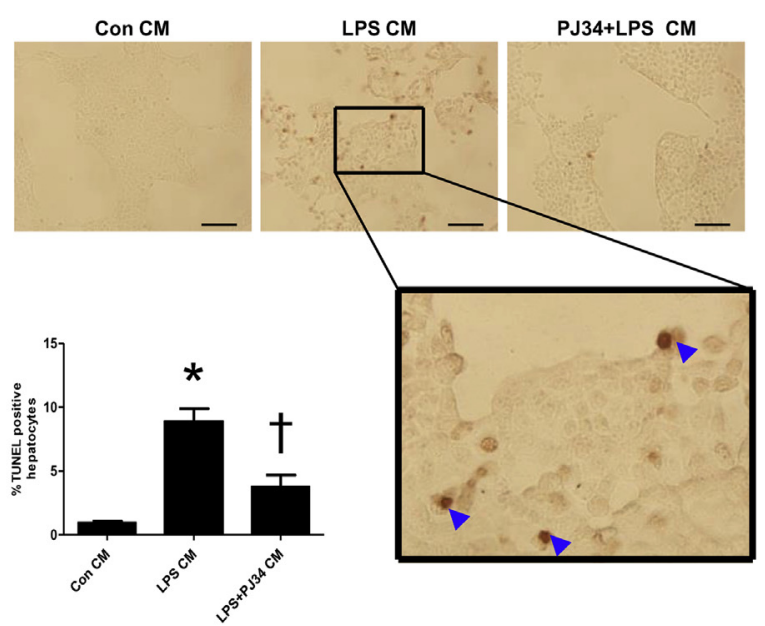

D

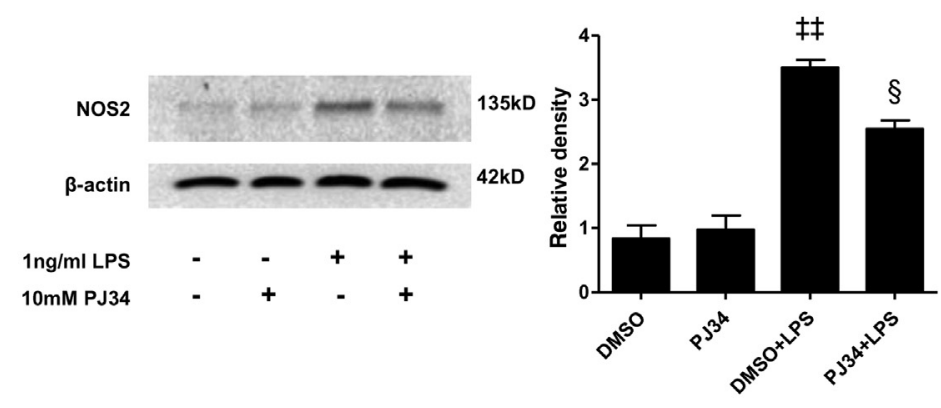

$\mathbf{F}$

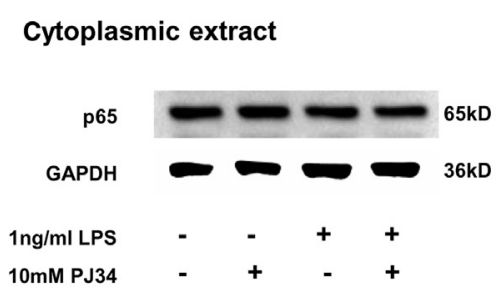

Nuclear extract

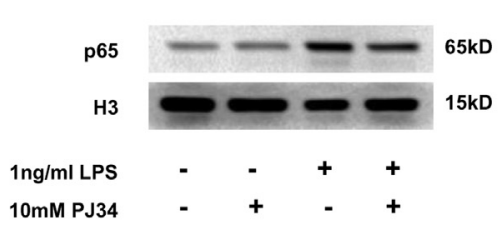

Cytoplasmic p65

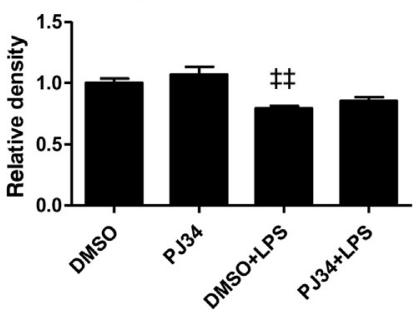

Nuclear p65

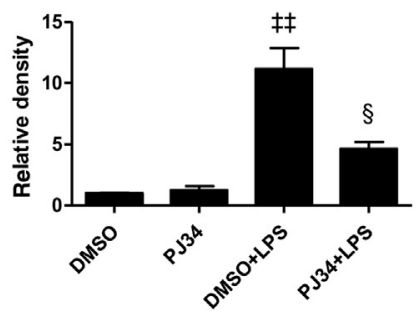

Figure 6 Poly (ADP-ribose) polymerase (PARP)-1 inhibition protects hepatocytes in vitro. Hepatocyte steatosis (A) and apoptosis (B) were evaluated by 0il Red 0 and TdT-mediated dUTP nick-end labeling (TUNEL) staining in ethanol (ETOH)-treated AML-12 hepatocytes incubated with supernatant collected from vehicle, lipopolysaccharide (LPS), or LPS and N-(6-oxo-5,6-dihydrophenanthridin-2-yl)-2-(N,N-dimethyno)tamide (PJ34) stimulated Raw264.7 cells, respectively. TUNEL-positive cells are indicated by blue arrowheads. C: Real-time RT-PCR analysis of Il6, Tnf $\alpha$, Mcp1, and Nos2 expression in $1 \mathrm{ng} / \mathrm{mL}$ of LPS stimulated Raw264.7 macrophages pretreated with PJ34 or vehicle. D: Western blot assay of Nos2 in LPS-stimulated Raw264.7 macrophages in different groups and statistical data from three independent experiments. E: Binding of NF- $\kappa$ B to specific element was detected by electromobility shift assay. F: Subcellular location of p65 were evaluated through Western blot in the left panel and calculated in the right panel. Three independent experiments were performed and analyzed. Original magnification: $\times 40(\mathbf{A}$ and $\mathbf{B})$. Scale bar $=50 \mu \mathrm{m}(\mathbf{A}$ and $\mathbf{B})$. ${ }^{*} P<0.05$ versus conditioned medium obtained from control Raw264.7 cells (Con CM); ${ }^{\dagger} P<0.05$ versus LPS-stimulated Raw264.7 cells (LPS CM) (A and B); ${ }^{\ddagger \ddagger} P<0.01,{ }^{\ddagger \ddagger \ddagger} P<0.001$ versus dimethylsulfoxide (DMS0); ${ }^{\S} P<0.05$, ${ }^{\S \S} P<0.01$ versus DMS0+LPS (C, D, and F). PJ34+LPS CM, LPS-stimulated PJ34 pretreated Raw264.7 cells. 
long-term ethanol consumption from severe injury. These protective effects were attributed to inhibiting $\mathrm{KC}$ activation and neutrophil infiltration, lipid metabolic regulation, and antiapoptosis effect.

During the past decades, numerous achievements about ALD have been made. Severity of liver injury in experimental ALD has been strongly correlated with increased portal vein endotoxin levels. ${ }^{34}$ An important mechanism by which alcoholic liver damage is caused is the activation of liver resident KCs. ${ }^{3}$ TLR4-dependent TNF- $\alpha$ signaling stimulated by gut-derived endotoxin plays a vital role in progression of ALD. ${ }^{21}$ Louvet et $\mathrm{al}^{6}$ and Wan et $\mathrm{al}^{8}$ found that limiting M1 KC polarization, accompanied with lower M1 gene mRNA levels, reduces alcohol-induced liver injury. They also found a negative correlation between the ratio of M2/M1 marker mRNA expression and liver triglyceride levels or serum transaminase. ${ }^{8}$ Another study reported that PARP inhibition reduces NO production by inhibiting NOS2 mRNA expression in activated macrophages induced by LPS. ${ }^{35}$ Besides, NOS2 is an important marker of the M1 phenotype macrophages. Therefore, we hypothesized that PARP1 participates in the progression of ALD by facilitating long-term ethanol consumption-induced $\mathrm{KC}$ activation.

In the present study, we reported that alcohol-induced elevation of serum ALT and liver triglyceride accumulation could be prevented by treatment of PARP inhibitor PJ34 or genetic deletion of PARP1. Hepatic steatosis, characterized by the accumulation of neutral fat in hepatocytes, is the earliest response of the liver to alcohol abuse. Previous studies have found that alcohol exposure, directly or indirectly, up-regulate the expression of sterol response element binding protein $1 \mathrm{c}$ and down-regulate the expression of PPAR $\alpha$, thus stimulating lipogenesis and inhibiting fatty acid oxidation. ${ }^{36-38}$ We found that ethanol-induced steatosis was markedly reduced in mice treated with PJ34 or PKO mice, as indicated by attenuated ethanol-induced liver lipid deposition. Our study also provides further insights into the role of PARP1 in ethanol-induced gene expression of lipid metabolism. It is well documented that mice treated with PJ34 or PKO mice exhibited decreased hepatic expression of lipogenic genes and increased level of lipidolytic genes. Our findings of decreased hepatic lipid deposition, lower LW/BW ratios, and limited $\mathrm{KC}$ activation in PKO mice suggest that hepatic lipid synthesis and decomposition balance could be influenced by hepatic inflammatory state. Erener et $\mathrm{al}^{39}$ observed an increased hepatic lipid deposition in C57BL/6 background PARP1 $1^{-1-}$ mice fed a high-fat diet, accompanied with increased LW/ BW ratios, limited lipid storage in adipocytes, and impaired glucose tolerance. However, these two mouse strains were created independently in two different backgrounds (SV129 and C57BL/6), and hepatic steatosis was induced through two different diets (ethanol feeding and high-fat diet). Whether such differences account for the contradicting effects of the PARPI deletion on lipid metabolism remains to be determined.
Excessive alcohol intake renders KCs hyperresponsive to endotoxin, thus resulting in enhanced production of TNF- $\alpha$ and other inflammatory cytokines. ${ }^{40}$ Recently, in the report from Louvet et $\mathrm{al},{ }^{6}$ inhibition of $\mathrm{KC}$ activation decreased hepatic M1 gene expression and reduced ethanol-induced hepatocyte steatosis. We found enhanced hepatic expression of M1 polarization marker genes (Il6, Tnfo, Monocyte chemotactic protein-1, and Nos2) in ethanol-fed mice and significant attenuation in mice treated by PJ34 or PKO mice. Further in vitro experimentation suggested that PARP activation facilitates LPS-induced NF- $\kappa$ B translocation to the nucleus in Raw264.7 macrophages. LCN2 drives neutrophilic infiltration and thus propagates the development of $\mathrm{ALD} .^{25} \mathrm{We}$ found that macrophage-derived LCN2 were profoundly enhanced on LPS stimulation and attenuated when treated with PJ34 in vitro, which might be the reason why neutrophil infiltration in alcoholic liver was limited in PKO mice or PJ34 treated mice. Collectively, limiting $\mathrm{KC}$ activation is sufficient to affect hepatic lipid balance, which might be an important protective pathway of PARP1 inhibition for treatment of alcoholic liver steatosis.

Apoptosis is one of the hallmarks of ethanol-induced liver injury. Long-term ethanol feeding increases hepatocellular apoptosis, and the extent of apoptosis is positively related to severity of liver injury. ${ }^{41}$ In the present study, PARP inhibition or PARP1 deficiency is efficient to protect hepatocytes from severe apoptosis induced by long-term ethanol feeding in vivo. In addition, limitation of macrophages activation induced by PJ34 administration is sufficient to decrease hepatic steatosis and apoptosis in vitro. Therefore, we can conclude that the antiapoptosis effect of PARP inhibitor is, at least in part, due to limited $\mathrm{KC}$ activation.

In conclusion, we identified that long-term ethanol consumption activates PARP1 in the liver, leading to KC activation, neutrophilic infiltration, hepatic steatosis, and apoptosis. Treatment with PARP inhibitor or PARP1 deficiency attenuated liver injury mainly through limiting $\mathrm{KC}$ activation in vivo and in vitro. These observations provide new insights into the mechanisms of ALD and significant implications for the development of novel strategies for the treatment of pathologic ALD by targeting PARP1.

\section{Acknowledgments}

We thank Dr. Wei Qian for the AML-12 hepatocyte cell line and cell culture technical support. We also thank veterinarian Shunchang Zhou for animal study technical support.

\section{Supplemental Data}

Supplemental material for this article can be found at http://dx.doi.org/10.1016/j.ajpath.2016.08.016. 


\section{References}

1. Gramenzi A, Caputo F, Biselli M, Kuria F, Loggi E, Andreone P, Bernardi M: Review article: alcoholic liver disease-pathophysiological aspects and risk factors. Aliment Pharmacol Ther 2006, 24:1151-1161

2. Mathurin P, Deng QG, Keshavarzian A, Choudhary S, Holmes EW, Tsukamoto H: Exacerbation of alcoholic liver injury by enteral endotoxin in rats. Hepatology 2000, 32:1008-1017

3. Maraslioglu M, Oppermann E, Blattner C, Weber R, Henrich D, Jobin C, Schleucher E, Marzi I, Lehnert M: Chronic ethanol feeding modulates inflammatory mediators, activation of nuclear factorkappaB, and responsiveness to endotoxin in murine Kupffer cells and circulating leukocytes. Mediators Inflamm 2014, 2014:808695

4. Koop DR, Klopfenstein B, Iimuro Y, Thurman RG: Gadolinium chloride blocks alcohol-dependent liver toxicity in rats treated chronically with intragastric alcohol despite the induction of CYP2E1. Mol Pharmacol 1997, 51:944-950

5. Lucey MR, Mathurin P, Morgan TR: Alcoholic hepatitis. N Engl J Med 2009, 360:2758-2769

6. Louvet A, Teixeira-Clerc F, Chobert MN, Deveaux V, Pavoine C, Zimmer A, Pecker F, Mallat A, Lotersztajn S: Cannabinoid CB2 receptors protect against alcoholic liver disease by regulating Kupffer cell polarization in mice. Hepatology 2011, 54:1217-1226

7. Wan J, Benkdane M, Alons E, Lotersztajn S, Pavoine C: M2 Kupffer cells promote hepatocyte senescence: an IL-6-dependent protective mechanism against alcoholic liver disease. Am J Pathol 2014, 184: $1763-1772$

8. Wan J, Benkdane M, Teixeira-Clerc F, Bonnafous S, Louvet A, Lafdil F, Pecker F, Tran A, Gual P, Mallat A, Lotersztajn S, Pavoine C: M2 Kupffer cells promote M1 Kupffer cell apoptosis: a protective mechanism against alcoholic and nonalcoholic fatty liver disease. Hepatology 2014, 59:130-142

9. Gibson BA, Kraus WL: New insights into the molecular and cellular functions of poly(ADP-ribose) and PARPs. Nat Rev Mol Cell Biol 2012, 13:411-424

10. Pacher P, Szabo C: Role of the peroxynitrite-poly(ADP-ribose) polymerase pathway in human disease. Am J Pathol 2008, 173:2-13

11. Kuhnle S, Nicotera P, Wendel A, Leist M: Prevention of endotoxin-induced lethality, but not of liver apoptosis in poly(ADP-ribose) polymerase-deficient mice. Biochem Biophys Res Commun 1999, 263:433-438

12. Oliver FJ, Menissier-de Murcia J, Nacci C, Decker P, Andriantsitohaina R, Muller S, de la Rubia G, Stoclet JC, de Murcia G: Resistance to endotoxic shock as a consequence of defective NFkappaB activation in poly (ADP-ribose) polymerase-1 deficient mice. EMBO J 1999, 18:4446-4454

13. Hassa PO, Hottiger MO: A role of poly (ADP-ribose) polymerase in NF-kappaB transcriptional activation. Biol Chem 1999, 380:953-959

14. Hassa PO, Covic M, Hasan S, Imhof R, Hottiger MO: The enzymatic and DNA binding activity of PARP-1 are not required for NF-kappa B coactivator function. J Biol Chem 2001, 276:45588-45597

15. Oumouna-Benachour K, Hans CP, Suzuki Y, Naura A, Datta R, Belmadani S, Fallon K, Woods C, Boulares AH: Poly(ADP-ribose) polymerase inhibition reduces atherosclerotic plaque size and promotes factors of plaque stability in apolipoprotein E-deficient mice: effects on macrophage recruitment, nuclear factor-kappaB nuclear translocation, and foam cell death. Circulation 2007, 115:2442-2450

16. Wang C, Zhang F, Wang L, Zhang Y, Li X, Huang K, Du M, Liu F, Huang S, Guan Y, Huang D, Huang K: Poly(ADP-ribose) polymerase 1 promotes oxidative-stress-induced liver cell death via suppressing farnesoid X receptor alpha. Mol Cell Biol 2013, 33: 4492-4503

17. Mukhopadhyay P, Rajesh M, Cao Z, Horvath B, Park O, Wang H, Erdelyi K, Holovac E, Wang Y, Liaudet L, Hamdaoui N, Lafdil F, Hasko G, Szabo C, Boulares AH, Gao B, Pacher P: Poly (ADP-ribose) polymerase-1 is a key mediator of liver inflammation and fibrosis. Hepatology 2014, 59:1998-2009
18. Hassa PO, Hottiger MO: The functional role of poly(ADP-ribose) polymerase 1 as novel coactivator of NF-kappaB in inflammatory disorders. Cell Mol Life Sci 2002, 59:1534-1553

19. Akinshola BE, Sharma S, Potter JJ, Mezey E: Ethanol enhances ADPribosylation of protein in rat hepatocytes. Hepatology 1992, 15: 471-476

20. Nomura F, Yaguchi M, Itoga And S, Noda M: Effects of chronic alcohol consumption on hepatic poly-ADP-ribosylation in the rat. Alcohol Clin Exp Res 2001, 25:35s-38s

21. Hritz I, Mandrekar P, Velayudham A, Catalano D, Dolganiuc A, Kodys K, Kurt-Jones E, Szabo G: The critical role of toll-like receptor (TLR) 4 in alcoholic liver disease is independent of the common TLR adapter MyD88. Hepatology 2008, 48:1224-1231

22. Zhou J, Zhai Y, Mu Y, Gong H, Uppal H, Toma D, Ren S, Evans RM, Xie W: A novel pregnane X receptor-mediated and sterol regulatory element-binding protein-independent lipogenic pathway. J Biol Chem 2006, 281:15013-15020

23. Souza MH, Troncon LE, Cunha FQ, Oliveira RB: Decreased gastric tone and delayed gastric emptying precede neutrophil infiltration and mucosal lesion formation in indomethacin-induced gastric damage in rats. Braz J Med Biol Res 2003, 36:1383-1390

24. Roychowdhury S, McMullen MR, Pisano SG, Liu X, Nagy LE: Absence of receptor interacting protein kinase 3 prevents ethanolinduced liver injury. Hepatology 2013, 57:1773-1783

25. Wieser V, Tymoszuk P, Adolph TE, Grander C, Grabherr F, Enrich B, Pfister A, Lichtmanegger L, Gerner R, Drach M, Moser P, Zoller H, Weiss G, Moschen AR, Theurl I, Tilg H: Lipocalin 2 drives neutrophilic inflammation in alcoholic liver disease. J Hepatol 2016, 64:872-880

26. Cohen JI, Roychowdhury S, DiBello PM, Jacobsen DW, Nagy LE: Exogenous thioredoxin prevents ethanol-induced oxidative damage and apoptosis in mouse liver. Hepatology 2009, 49:1709-1717

27. Pastorino JG, Shulga N, Hoek JB: TNF-alpha-induced cell death in ethanol-exposed cells depends on p38 MAPK signaling but is independent of Bid and caspase-8. Am J Physiol Gastrointest Liver Physiol 2003, 285:G503-G516

28. McVicker BL, Tuma DJ, Kubik JL, Tuma PL, Casey CA: Ethanolinduced apoptosis in polarized hepatic cells possibly through regulation of the Fas pathway. Alcohol Clin Exp Res 2006, 30:1906-1915

29. Masutani M, Nakagama H, Sugimura T: Poly(ADP-ribosyl)ation in relation to cancer and autoimmune disease. Cell Mol Life Sci 2005, 62: 769-783

30. Mota RA, Sanchez-Bueno F, Saenz L, Hernandez-Espinosa D, Jimeno J, Tornel PL, Martinez-Torrano A, Ramirez P, Parrilla P, Yelamos J: Inhibition of poly(ADP-ribose) polymerase attenuates the severity of acute pancreatitis and associated lung injury. Lab Invest 2005, 85:1250-1262

31. Pacher P, Szabo C: Role of poly(ADP-ribose) polymerase 1 (PARP-1) in cardiovascular diseases: the therapeutic potential of PARP inhibitors. Cardiovasc Drug Rev 2007, 25:235-260

32. Shevalye H, Stavniichuk R, Xu W, Zhang J, Lupachyk S, Maksimchyk Y, Drel VR, Floyd EZ, Slusher B, Obrosova IG: Poly(ADP-ribose) polymerase (PARP) inhibition counteracts multiple manifestations of kidney disease in long-term streptozotocin-diabetic rat model. Biochem Pharmacol 2010, 79:1007-1014

33. Underhill C, Toulmonde M, Bonnefoi H: A review of PARP inhibitors: from bench to bedside. Ann Oncol 2011, 22:268-279

34. Nanji AA, Khettry U, Sadrzadeh SM, Yamanaka T: Severity of liver injury in experimental alcoholic liver disease: correlation with plasma endotoxin, prostaglandin E2, leukotriene B4, and thromboxane B2. Am J Pathol 1993, 142:367-373

35. Virag L, Szabo C: Purines inhibit poly(ADP-ribose) polymerase activation and modulate oxidant-induced cell death. FASEB J 2001, 15: 99-107

36. You M, Fischer M, Deeg MA, Crabb DW: Ethanol induces fatty acid synthesis pathways by activation of sterol regulatory element-binding protein (SREBP). J Biol Chem 2002, 277:29342-29347 
37. Yu S, Rao S, Reddy JK: Peroxisome proliferator-activated receptors, fatty acid oxidation, steatohepatitis and hepatocarcinogenesis. Curr Mol Med 2003, 3:561-572

38. Wagner M, Zollner G, Trauner M: Nuclear receptors in liver disease. Hepatology 2011, 53:1023-1034

39. Erener S, Mirsaidi A, Hesse M, Tiaden AN, Ellingsgaard $H$, Kostadinova R, Donath MY, Richards PJ, Hottiger MO: ARTD1 deletion causes increased hepatic lipid accumulation in mice fed a high-fat diet and impairs adipocyte function and differentiation. FASEB J 2012, 26:2631-2638

40. Nath B, Szabo G: Alcohol-induced modulation of signaling pathways in liver parenchymal and nonparenchymal cells: implications for immunity. Semin Liver Dis 2009, 29:166-177

41. Higuchi H, Kurose I, Kato S, Miura S, Ishii H: Ethanol-induced apoptosis and oxidative stress in hepatocytes. Alcohol Clin Exp Res 1996, 20:340a-346a 